\title{
Cost of operating a nuclear magnetic resonance imaging system
}

\author{
G R CHERRYMAN
}

Nuclear magnetic resonance is a new, exciting, and expensive method of medical imaging. Increasing clinical and public awareness of the technique is encouraging radiologists and others to consider the costs of installing and running nuclear magnetic resonance units. The intending purchaser is faced with a choice between the cheaper and easier to install imager, based around a low field strength resistive magnet, or the more expensive and difficult to shield superconducting system, with some improvement in resultant spatial resolution. In Britain, given the present economic constraints, the practiral difficulty and expense of installing superconducting systems into existing hospital buildings, as well as the generally unproved advantage of operating at these higher magnetic field strengths, most nuclear magnetic resonance installations have been based around resistive systems and this pattern is likely to remain in future. Superconducting systems will probably be limited to teaching centres with a special interest in research and perhaps certain specialist clinical disciplines.

The first nuclear magnetic resonance imager in Aberdeen was built by and installed in the university department of biomedical physics and bioengineering. In 1983 a second machine was built by the same department and installed in a purpose built extension to the nuclear medicine wing in the Royal Infirmary. The running costs of this machine are met by the Medical Research Council as part of a joint project with the Department of Health and Social Security into the clinical value of nuclear magnetic resonance imaging. Five clear areas of research interest have been defined: intracranial imaging (both neurological and psychiatric), head and neck tumours, liver disease, pancreatic disease, and gynaecological cancer. For the remainder of its time the scanner is used for routine clinical referrals. Some machine time is given back to the physicists for research and development.

The patient workload and running costs of our imager are similar to those that might be expected in a commercial installation. There are obvious cost and efficiency advantages in having an imager built on site and maintained by the department of biomedical physics and bioengineering. Would be purchasers of nuclear magnetic resonance equipment are faced with paying a commercial price for the machine and high annual maintenance service contracts.

The patient workload and costs of running our imager for one year are presented. Projections of running costs that might be expected in commercial installations are made. Nuclear magnetic resonance is a rapidly developing imaging technique. Changes that might be expected in the future are discussed in terms of their effect on running costs. The probable impact on a radiological department is also briefly discussed.

\section{Materials and methods}

The Aberdeen mark II imager was designed and built by the university. It is based around a four coil, air cored, water cooled resistive magnet operating at 0.08 tesla ( 800 gauss). The resultant imaging frequency for hydrogen protons is $3.4 \mathrm{MHz}$. The magnet is mounted vertically and the patient lies supine on a mobile couch within the body coil (diameter $45 \mathrm{~cm}$ ). The

Department of Biomedical Physics and Bioengineering, University of Aberdeen, Aberdeen AB9 2ZD

G R CHERRYMAN, MB, FRCR, senior medical research fellow and honorary consultant radiologist direction of the static field is in the $\mathrm{Z}$ direction-that is, posterior/anterior in the patient. A separate and easily detached head coil (diameter $25 \mathrm{~cm}$ ) is used for head and neck imaging. The details of the machine and the principles of spin warp imaging are discussed elsewhere. ${ }^{22} \mathrm{~A}$ relatively fixed pulse sequence is used. At present all routine imaging is undertaken at a tau value of $200 \mathrm{~m} / \mathrm{s}$. On occasions extra scans, at different tau values, will be obtained.

The resolution of any scanner depends on the chosen pixel size and slice thickness. We are still imaging on a $128 \times 128$ matrix; this gives a resultant pixel size of $3.5 \times 3.5 \mathrm{~mm}$ for the body coil and $1.9 \times 1.9 \mathrm{~mm}$ for the head coil. Slice thickness with the body coil is $16 \mathrm{~mm}$ and with the head coil $12 \mathrm{~mm}$. The information for each section is acquired in $256 \mathrm{~s}$ and after processing may be displayed in four modes: proton density (S1), inversion recovery (S2), subtracted (S1-S2), and as a calculated T1 image. The patient may be examined in the sagittal, coronal, or axial plane. The sagittal section is midline with the patient centred to the midline of the coil. Offset sagittal sections are obtained only by moving the patient inside the coil and are limited by the diameter of the coil and the size of the patient. Similarly, the coronal section is again in a fixed plane and offset imaging is limited. The patient has to be removed from the imager and couch, the number of mattresses on the couch adjusted, and the patient then repositioned inside the imager. We usually obtain sagittal and coronal images only when the preset imaging planes are likely to prove rewarding. Axial increments for our transverse scans are obtained by manually moving the couch through the imager.

Image data are stored on floppy discs. At least one hour of the radiographer's day is set aside for making the "hard copy" photographic images. The system may be run single handed by an experienced radiographer, but patient throughput, safety, and confidence are greatly increased by having an assistant to change patients, watch the patients inside the imager, and help with the documentation and archiving. Any installation would need at least two radiographers trained in nuclear magnetic resonance techniques to provide holiday and emergency cover. The limited scan and pulse sequence options at present available on our machine reduce the need for full time radiological presence.

As with any scanning unit facilities are best used with an efficient booking system, radiological monitoring of requests, reliable portering, and good documentation. We have found a secretary/research assistant indispensable. The reporting of scans is time consuming and a separate reporting and display console is essential.

We have kept detailed records of our workload over the past 12 months ( 1 April 1984 to 1 April 1985) and this has been used as the basis for a retrospective analysis.

\section{Results}

During the year under review the scanner was available from $9 \mathrm{am}$ till $5 \mathrm{pm}$, Monday to Friday. There were 230 designated working days in this period and the scanner worked $220(95 \%)$ of these. The 10 missing days were caused by minor faults in the computer and one of the gradient amplifiers. The machine was made available to the biomedical physics department for 20 days of the year as part of a planned programme of improvement; the remaining 12 weekdays were holidays. A total of 1517 patients and volunteers were examined. Thirty one per cent (473) were intracranial examinations and $69 \%$ (1048) examinations of some other part of the body. The average daily workload was 6.9 patients, and 11430 sections were obtained at an average of 7.5 per patient and 51.75 a day. Each patient thus took an average time of 72 minutes, of which only 32 minutes (44\%) were spent being scanned.

The cost of scanning these patients has been itemised in table I. The average cost per patient was $£ 49 \cdot 49$. The imager is maintained 
by the university and no service contract for the imager is required. There are service contracts on the computer and some other electrical items. No major repairs or replacements were required during this year. The cost of electricity and water has been estimated and calculated at industrial rates.

A trained and reliable radiographer is obviously essential. We have another trained and experienced radiographer available in the department for holiday and emergency cover. A staff nurse on a weekly rota from the nuclear medicine department is used for patient help. A full time research oriented radiologist is essential for the correct organisation and monitoring of our clinical trials. The help of a physicist is also required for the testing of our machine and quality control. We have the full support of our nuclear medicine physicists. We have included the cost of a half time physicist in our calculations; the remainder of his time is spent in nuclear medicine. All salaries are quoted at $125 \%$ to include the cost of superannuation and National Insurance.

The baseline cost of a nuclear magnetic resonance examination on our system, during the period of accounting, was thus $£ 49.49$, of which $£ 40 \cdot 05(80 \%)$ covered staff costs. This figure does not include any amount to cover the cost of research and development or the buying, building, and installing of our imager.

\section{Discussion}

The Aberdeen nuclear magnetic resonance imager is at present heavily committed to research. This makes demands on radiological and secretarial time much greater than might be expected in a typical clinical service installation. A basic nuclear magnetic resonance facility could be provided using a single radiographer and a part time radiologist. This assumes, of course, that the installation has access to a radiological department for secretarial help, the occasional nursing assistance required, and the provision of a second trained radiographer to provide radiographic cover. Nonresearch installations may also save on floppy discs. There would be no necessity to maintain digital information longer than six weeks. This would reduce the annual floppy disc budget by approximately $£ 2800$. Table II shows the projected annual costs for both the basic nuclear magnetic resonance facility and a clinical nuclear magnetic resonance unit. The limitations on radiological, secretarial, and physics help should ensure that apart from basic clinical imaging research little could be undertaken.

When considering commercial machine installations, possible savings in unit staffing are offset against the higher cost of annual maintenance. A full service contract for the machine is required. Promotional literature for a resistive nuclear magnetic resonance system similar to our own has estimated this at $\$ 20000$ a year. This represents approximately $6 \%$ of the purchase price. Manufacturers in general have emphasised the ease of maintenance and reduced running costs when nuclear magnetic resonance units are compared with $x$ ray computed tomography units, but the market for nuclear magnetic resonance imagers is limited and competitive. It might be that manufacturers will increase their annual maintenance contracts to $8 \%$ or $10 \%$ of the purchase price in order to maintain a realistic purchase price and yet recover research and development costs.

The cost of nuclear magnetic resonance images varies enormously. A basic low field resistive system might cost from $£ 350000$ upwards. Superconducting magnet systems with high fields and the possibility of spectroscopy will cost between $£ 1$ million and $£ 2$ million. The purchaser must also consider the cost of installation. Small resistive systems may be installed in existing rooms, often at acceptable prices ( $£ 10000$ upwards). The cost of installing the superconducting magnet system is proportionally greater and in some instances may exceed the cost of the imager. The efficiency of our imager is due partly to the simplicity of our pulse sequence. Technical development in nuclear magnetic resonance has tended to increase the choice of imaging options and in turn this makes greater demand on radiological time. There is no doubt that the clinical efficiency of a nuclear magnetic resonance unit (as with $x$ ray computed tomography units) is greatly improved by the full time presence of a radiologist. The increasing number of pulse sequence and scan options also increases the examination time. It is likely that even with multislice acquisition the number of patients that can be examined in a standard working day cannot be greatly improved.

The clinical usefulness of nuclear magnetic resonance imaging has yet to be fully determined. This makes it difficult to estimate savings that might accrue from the reduction in demand for other imaging techniques and radiological examinations. There are at

TABLE I-Running costs, 1 April 1984 to 1 A pril 1985

\begin{tabular}{|c|c|c|}
\hline & $\begin{array}{l}\text { Salary } \\
(\mathfrak{L})\end{array}$ & $\begin{array}{c}\text { Salary }+25 \% \\
\text { (£) }\end{array}$ \\
\hline \multicolumn{3}{|l|}{ Staff: } \\
\hline Radiologist & 26080 & 32600 \\
\hline Radiographer & 6000 & 7500 \\
\hline Staff nurse & 6582 & 8227 \\
\hline Secretary & 4692 & 5865 \\
\hline \multirow[t]{2}{*}{ Physicist (half) } & 5250 & 6562 \\
\hline & 48604 & 60754 \\
\hline \multicolumn{3}{|l|}{ Expenses: } \\
\hline 100 floppy discs $(a) 33.50$ & 3500 & \\
\hline 4551 films (a) $40 \mathrm{p}$ & 1020 & \\
\hline Stationery & 250 & \\
\hline Power $@ 31 \mathrm{p} /$ unit & 2091 & \\
\hline Water (3696000 litres) & 465 & \\
\hline \multirow[t]{2}{*}{ Service contracts } & 7000 & \\
\hline & 14326 & 14326 \\
\hline Total & & 75080 \\
\hline
\end{tabular}

TABLE II-Projected annual running costs for a service nuclear magnetic resonance imager

\begin{tabular}{|c|c|c|c|}
\hline & $\begin{array}{l}\text { Basic facility } \\
(£)\end{array}$ & \multicolumn{2}{|c|}{$\begin{array}{c}\text { Clinical unit } \\
(£)\end{array}$} \\
\hline Radiologist & & & \\
\hline 5 sessions & 16300 & 16300 & \\
\hline 10 sessions & & & 32600 \\
\hline Radiographer: & & & \\
\hline One & 7500 & & \\
\hline Two & & 15000 & 15000 \\
\hline Secretary (half time) & & 2933 & 2933 \\
\hline Staff costs & 23800 & 34233 & 50533 \\
\hline Service contract (at $6 \%$ ) & 20000 & 20000 & 20000 \\
\hline Data storage discs & 700 & 700 & 700 \\
\hline Other costs as in table I & 3826 & 3826 & 3826 \\
\hline Operating costs & 24526 & 24526 & 24526 \\
\hline Total & 48326 & 58759 & 75059 \\
\hline Cost per patient & $31 \cdot 86$ & $38 \cdot 73$ & $49 \cdot 47$ \\
\hline
\end{tabular}

present few areas where our results with nuclear magnetic resonance have suggested that it will completely replace other radiological techniques. In many instances nuclear magnetic resonance is likely to emerge as another alternative investigation, useful in certain $\mathrm{N}$ cases. Within the next few years the addition of nuclear magnetic resonance to any radiology department is unlikely to reduce greatly the workload and budget of the remainder of that department.

I should like to thank Mr G Crosher and Miss L Murray for radiographic and secretarial help; the Medical Research Council for funding the Aberdeen clinical evaluation of nuclear magnetic resonance imaging programme; and the University of Aberdeen's department of biomedical physics and bioengineering.

\section{References}

1 Edelstein WA, Hutchison JMS, Johnson G. Spin-warp NMR imaging and applications to human whole-body imaging. Phys Med Biol 1980;25:751-6.

2 Hutchison JMS, Edelstein WA, Johnson G. A whole-body NMR imaging machine. $\mathcal{f}$ Phys [E] 1980;13:947-55.

(Accepled 12 June 1985)

\section{무} \)

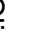

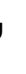

